\title{
Comparison of lung ultrasonography findings with chest computed tomography results in coronavirus (COVID-19) pneumonia
}

\author{
Korgün Ökmen ${ }^{1}$ [D $\cdot$ Durdu Kahraman Yıldız ${ }^{1}$ Emel Soyaslan ${ }^{1}$
}

Received: 25 January 2021 / Accepted: 8 February 2021 / Published online: 26 February 2021

(c) The Japan Society of Ultrasonics in Medicine 2021

\begin{abstract}
Purpose The purpose of our study was to determine the usability of lung ultrasonography (LUS) in the diagnosis of COVID19 , and to match the morphological features of lesions detected on computed tomography (CT) with the findings observed on LUS.

Methods Sixty patients with COVID-19 were included in this prospective study. Patients were examined by radiology and anesthesia clinic specialists for a visual CT score. A LUS 12-zone ultrasonography protocol was applied by the investigator blinded to the CT and PCR test results. The characteristics of abnormal findings and the relationship of lesions to the pleura and the distance to the pleura were investigated.

Results Forty-five males and 25 females evaluated within the scope of the study had an average age of $61.2 \pm 15.3$ years. The total CT score was calculated as $14.3 \pm 5.3$, and the LUS score was found to be $19.9 \pm 7.6$. There was a statistically significant positive correlation between the measured LUS and CT scores $(r=0.857, p<0.001)$. The mean distance of these lesions to the pleura was $5.2 \pm 1.76 \mathrm{~cm}$. LUS findings in 51 areas corresponded to non-pleural lesions on CT. There was a negative correlation between the measured distance to the pleura and the LUS scores $(p<0.001, r=-0.708)$.

Conclusion The results of this study showed that the correlation between CT and LUS findings may be used in the diagnosis of COVID-19 pneumonia, although there are some limitations.

ClinicalTrials.gov identifier: NCT04719234.
\end{abstract}

Keywords COVID-19 $\cdot$ Lung ultrasound $\cdot$ Computed tomography $\cdot$ Pneumonia

\section{Introduction}

With the recognition of SARS-CoV-2 infection as a pandemic infection by the World Health Organization (WHO), many countries began to work on the diagnosis and treatment of this disease [1].

The coronavirus, which is recognized as the cause of this infection, can be transmitted via droplets and progress to severe acute respiratory distress syndrome (ARDS), sepsis, and septic shock, which may require intensive care in patients with comorbidities by reaching a life-threatening dimension from a mild upper respiratory tract infection [2].

Korgün Ökmen

korgunokmen@gmail.com

1 Department of Anesthesiology and Reanimation, Bursa Yuksek Ihtisas Training and Research Hospital, University of Health Sciences, Mimar Sinan Mah. Emniyet Street Yıldırım, Bursa, Turkey
During the pandemic period, the effect of this coronavirusrelated disease on the respiratory tract and the observation of pulmonary findings in the foreground have required greater use of high-resolution computed tomography (HR-CT) [3]. The reliability of CT used in thoracic imaging was found to be $97 \%$ compared to the PCR test. Ground-glass opacities (GGO), crazy paving pattern, and interlobular septal thickening are the main findings identified on CT [4, 5]. Even though HR-CT is accepted as the gold standard imaging technique, the difficulty of moving patients being treated in intensive care units, the use of radiation, and the application of different treatments (mechanical ventilation or even extracorporeal membrane oxygenation, etc.) in ICU treatments limit its re-application [6]. In many studies in the literature, it was shown before the COVID-19 pandemic that lung ultrasonography (LUS) could be used for diagnosis and followup by many departments such as intensive care units and emergency departments [7, 8]. Considering the prevalence of pneumonia and the emergence of a condition like ARDS 
caused by COVID-19 in the lungs along with the disadvantages of CT, it started to be used more often in the diagnosis and follow-up of COVID-19. Even though LUS findings of the disease have similarities with viral pneumonia, they also have some differences. Pleural irregularity, abnormalities in B lines, and fused B lines are some of the LUS findings that have been found in COVID-19 patients. Studies have reached conclusions about the advantages of LUS and areas where it may be limited [9].

The aim of this study was to determine the usability of LUS in the diagnosis of COVID-19, and to match the morphological features of lesions observed on CT with the abnormal findings identified on LUS.

\section{Materials and methods}

\section{Patients}

Following the ethics committee's approval, 60 patients who were hospitalized in the intensive care unit with a diagnosis of COVID-19 pneumonia were evaluated within the scope of this prospective study (Ethical number 2011-KAEK-25 2020/05-21). Patients between 18 and 85 years of age, who were hospitalized in the intensive care unit with the suspicion of COVID-19 and who had a PCR test and thoracic CT, were included in the study. Patients with previous lung and thoracic wall surgery, anatomically having thoracic wall abnormalities, and who did not consent to participate in the study were not included in the study.

\section{Interventions}

\section{CT procedure}

HR-CTs were performed with a 128-row scanner (SOMATOM Definition AS+; Siemens Healthineers, Erlangen, Germany) in the supine position during patient inspiration. The evaluation parameters of all the HR-CT scans with the 128-row scanner were as follows: $120 \mathrm{kVp}$, 81 reference mAs. Reconstruction parameters for the lung images were as follows: slice thickness $3 \mathrm{~mm}$, sharp reconstruction algorithm (3.0 B157 and lung window width, 1,200 HU; level, - $600 \mathrm{HU})$. Reconstruction parameters for mediastinal images were as follows: slice thickness $5.0 \mathrm{~mm}$, reconstruction algorithm (5.0 Bf37), and mediastinal window (width, $350 \mathrm{HU}$; level, $350 \mathrm{HU}$ ). Thoracic CT was performed before the intensive care hospitalization with the indication of the emergency department, and was reported and scored by the radiology department, which was unaware of the lung ultrasonography, in company with the staff of the anesthesiology and reanimation clinic
(DKY and ES). For the scoring method, the validated thinsection CT examination method of Chang et al. was used [10].

Each of the five lobes of the lung was scored in terms of involvement of ground-glass opacities, consolidation, interstitial opacity, and air trapping: $0 \%$ (0 points), $1-5 \%$ (1 point), 5-25\% (2 points), 25-50\% (3 points), $50-75 \%$ (4 points), or $>75 \%$ ( 5 points). Values ranging between 0 and 25 were recorded for each lung lobe and as total CT score data [11].

Moreover, the COVID-19 Reporting and Data System (CO-RADS), which is a categorical evaluation schema for pulmonary involvement of COVID-19 on non-contrast chest $\mathrm{CT}$, was used, and $\mathrm{CT}$ results were recorded in the areas defined for the LUS scoring system [12, 13].

\section{LUS protocol}

Ultrasonography evaluations were carried out by a researcher who was experienced in LUS and performing ultrasonography, and the evaluation was recorded with the convex ultrasound probe $(2-6 \mathrm{MHz})$. The investigator was blinded to patient information such as medical history, PCR test result, laboratory measurements, and CT scan results.

LUS was performed within a maximum of $6 \mathrm{~h}$ of the patient's hospitalization in the intensive care unit. Examinations in the intensive care unit, which was allocated to infection, were performed after all the protective precautions were taken. Together with the 12-area protocol as 6 areas for the right lung and 6 areas for the left lung, lung areas were separated into six areas for convenient scanning: anterior-superior (upper part of the internipple line in the midclavicular line), anterior-inferior (lower part of the internipple line in the midclavicular line), middle-superior (upper part of the internipple line above the midaxillary line), middle-inferior (upper part of the internipple line above the midaxillary line), rear-superior (above the line joining the lower ends of the scapula in the paravertebral line), and posterior-inferior (below the line joining the lower ends of the scapula in the paravertebral line) [12].

All the abnormal findings, primarily pleural line abnormalities, B lines, consolidations, and pleural effusion, were recorded in each chest area examination. A scoring system was used for each area. Scores ranged from 0 to 3 (0 points: B lines, including A lines and $<3,1$ point: less than $50 \%$ of the intercostal space combined B line, 2 points: B lines covering more than $50 \%$ of the intercostal space (white lung), 3 points: recorded as consolidation or pleural effusion). A score of $0-36$ points in total was collected for $0-18$ points for right and left lung [13]. 


\section{Evaluation parameters}

\section{Outcome measures}

The primary outcome measure was to investigate the relationship between thoracic CT imaging results and LUS results. Accordingly, visual CT scores were used for thoracic CT images, and the visual LUS score algorithm was used for LUS. The secondary outcome measures were to identify the characteristics of the abnormal findings detected on thoracic CT and LUS, the relation of the lesions with the pleura, and their distance to the pleura.

\section{Statistical analysis}

SPSS 22.0 statistical software was used for data analysis. Data were presented as mean \pm standard deviation, and the quantitative data were presented as the percentage. The Shapiro-Wilk test was used to evaluate whether or not the data were normally distributed. Student's $t$ test was used to compare normally distributed data. Spearman's rho correlation test was used to evaluate the correlation between the LUS score and the CT score. In cases where the $P$ value was found to be less than 0.05 , the relationship was considered significant.

\section{Power analysis}

In our pilot study, we determined the correlation value between CT scores and LUS scores as $r$ : 0.339. The necessary sample size was determined as 60 patients to obtain an $85 \%$ study power.

\section{Results}

Sixty patients (45 males and 25 females) evaluated within the scope of the study had an average age of $61.2 \pm 15.3$ years. Other descriptive data of patients are presented in Table 1. All patients were diagnosed with COVID-19 based on PCR test and CT and clinical findings before hospitalization. Forty-two patients were classified as CO-RADS 5, and 18 patients were classified as CO-RADS 4. Bilateral lung involvement was observed in 54 patients based on examination of the thorax tomography data, and single lung involvement was detected in six patients (four and two patients with isolated right and left lung involvement, respectively).

The most common lesions on CT images were groundglass opacities, crazy paving sign, and consolidation (Table 2) (Fig. 1). The mean LUS score was found to be $19.9 \pm 7.6$, and the most common findings were pleural line abnormalities and B line abnormalities (Table 2) (Fig. 2). The total CT score was $14.3 \pm 5.3$, and CT scores
Table 1 Comparison of the demographic characteristics of the patients ( $n: 60)$

\begin{tabular}{ll}
\hline Age (years) & $61.2 \pm 17.3$ \\
BMI $\left(\mathrm{kg} / \mathrm{m}^{2}\right)$ & $26.3 \pm 5.25$ \\
Gender & \\
M/F & $45(\% 75) / 25(\% 25)$ \\
Apache score & $24.4 \pm 5.6$ \\
Smoke \pm & $25(\% 41) / 35(\% 58)$ \\
Comorbidity & \\
No comorbidities & 12 \\
Hypertension & 24 \\
Diabetes & 14 \\
Cardiovascular disease & 13 \\
Renal disease & 1 \\
COPD & 7 \\
Cancer & 4 \\
Other & 8 \\
\hline
\end{tabular}

Mean \pm SD

$B M I$ body mass index, $M$ male, $F$ female, $C O P D$ chronic obstructive pulmonary disease

Table 2 CT and LUS findings

\begin{tabular}{lll}
\hline CT findings & Right $(n=58)$ & Left $(n=56)$ \\
\hline Ground-glass opacities & $58(\% 100)$ & $56(\% 100)$ \\
Crazy paving sign & $24(\% 41)$ & $20(\% 35)$ \\
Subpleural lines, & $8(\% 13)$ & $10(\% 17)$ \\
Consolidation & $8(\% 13)$ & $8(\% 14)$ \\
Pleural effusion & $2(\% 3.4)$ & $1(\% 1.7)$ \\
LUS findings & & \\
Irregular pleural line & $58(\% 100)$ & $56(\% 100)$ \\
B lines & & \\
Compound B lines (less than & $23(\% 39.6)$ & $22(\% 39.2)$ \\
$\quad 50 \%$ of the intercostal space) & & 30 \\
$\quad$ Diffuse B lines (white lung) & $28(\% 39.6)$ & $12(\% 21.4)$ \\
$\quad$ Subpleural consolidation & $14(\% 24)$ & $1(\% 1.7)$ \\
Pleural effusion & $2(\% 3.4)$ & $3(\% 5.3)$ \\
Consolidation & $1(\% 1.7)$ & \\
\hline
\end{tabular}

categorized by right- and left-side values and lung segments are presented in Table 3.

There was a statistically significant positive correlation between the measured LUS and CT scores $(r=0.857$, $p<0.001)$. When the right and left lung scores were evaluated separately, no correlation was found between the right LUS and CT scores (Table 3).

The CT scores of the right upper lobe $(1.36 \pm 1.08)$ and the left upper lobe $(1.75 \pm 1.37)$, which were calculated according to the lung lobes, were statistically significantly lower when compared to the other lobes (Fig. 3). 
Fig. 1 Thoracic CT images of COVID-19-related pneumonia. a Black arrows: halo sign, b non-pleural lesion and distance to the pleura, $\mathbf{c}$ white arrows: ground-glass opacities, $\mathbf{d}$ nonpleural hilar lesion and distance to the pleura

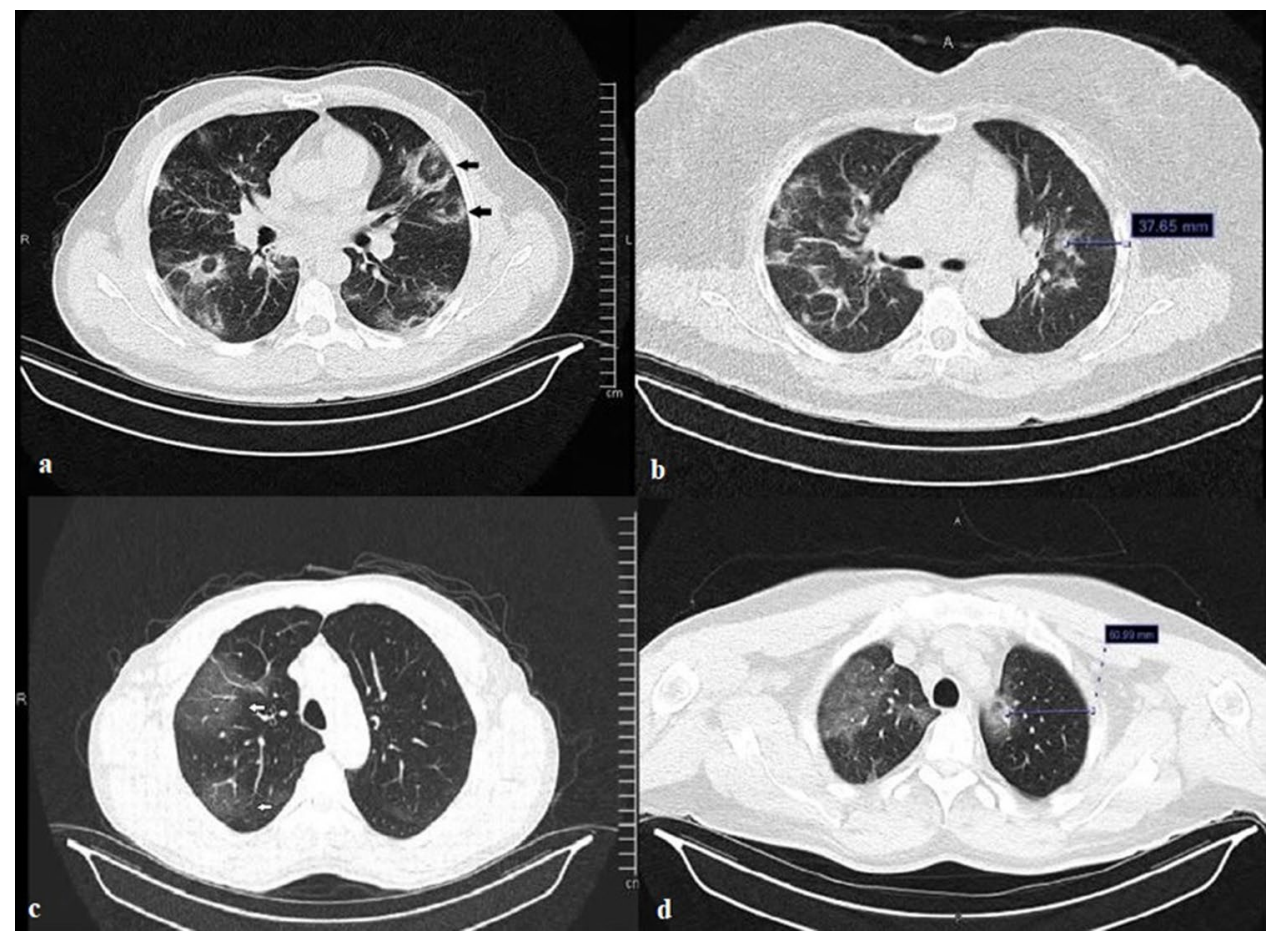

A comparison of the most frequently detected lesions on CT and LUS findings detected in the same region in the patients evaluated are presented in Table 4.

When the relation of the lesions detected in the areas scanned on CT with the pleura was evaluated, lesions that were not related to the pleural were found in 51 areas $(6.9 \%)$ of a total of 720 areas examined. The mean distance of these lesions to the pleura was $5.2 \pm 1.76 \mathrm{~cm}$. LUS findings in the 51 regions with non-pleural lesions on CT are presented in Table 4 . There was a negative correlation between the measured distance to the pleura and the LUS scores $(p<0.001$, $r=-0.708$ ) (Fig. 4).

\section{Discussion}

The results of this study showed that the scores obtained with LUS were highly consistent with the scores obtained by thoracic CT evaluation. It was revealed that the groundglass appearance detected on CT imaging could be displayed as different findings such as different B lines and pleural line abnormalities on LUS. Furthermore, it was shown that there might be limitations in detecting non-pleural lesions detected on CT via LUS.

LUS has been used in different types of lung diseases, including pneumonia, for a long time. In their study comparing thoracic CT results, which are considered to be the gold standard, and LUS results, Lichtenstein et al. evaluated 118 lungs and found that the sensitivity and specificity of LUS were 90 and $98 \%$, respectively. In their study, in which alveolar consolidations were evaluated, it was reported that LUS was poor at detecting small lesions that did not reach the pleura [14]. In two different studies carried out with patients diagnosed with community-acquired pneumonia, 211 patients and 342 patients were evaluated. While sensitivity and specificity were found to be above $95 \%$, lesions detected by other radiological methods were not identified by LUS in $8 \%$ of lesions in both studies $[15,16]$. There are also studies in the literature on how lesions detected on chest tomography appear on LUS. In those studies, it was reported that different lesions were detected on LUS in patients who had diffuse ground-glass opacification on tomography; however, there were more B line abnormalities [17]. Other study results indicate that ground-glass opacities in patients diagnosed with viral pneumonia can predominantly be observed as pleural line abnormalities on LUS [18].

The high transmission rate of COVID-19 and the hospitalization of many COVID-19 cases have led to an increased burden on hospitals. Even though thoracic CT has a high diagnostic value, its disadvantages in practice have increasingly necessitated the use of LUS. Researchers have investigated the usefulness and accuracy of LUS in the diagnosis and follow-up of COVID-19 with different types of studies [9]. In their study, Volpicelli et al. obtained image results of COVID-19 pneumonia, such as B line abnormalities and irregular or fragmented pleural lines, similar to those detected in other types of pneumonias. The two findings that they thought might be specific to COVID-19 pneumonia were combined B lines, which they referred to as a "light beam" forming a multiform vertical artifact and vertical 


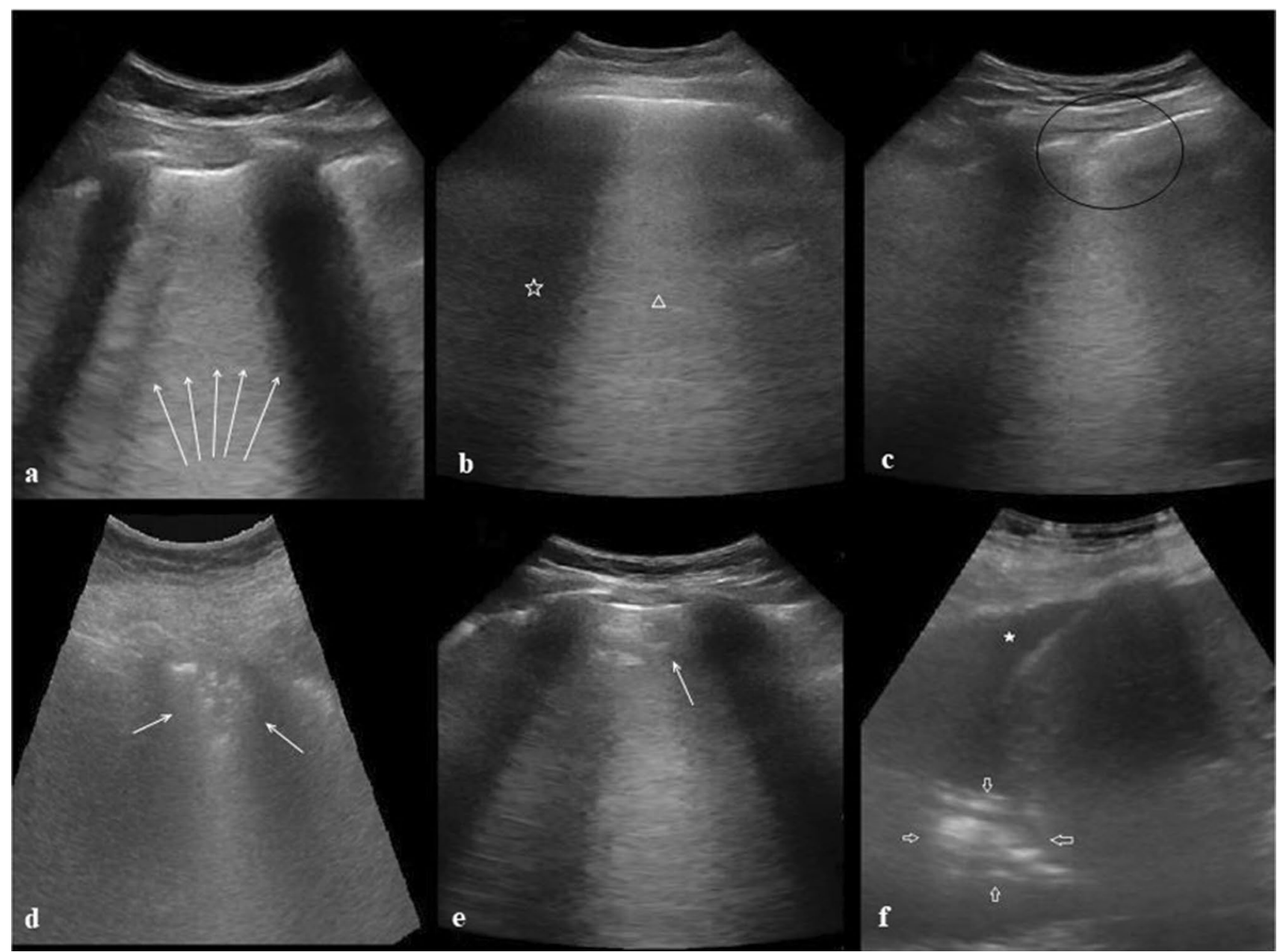

Fig. 2 LUS images of COVID-19-related pneumonia. a White arrows: diffuse B lines, $\mathbf{b}$ white star: lesion-free zone, white triangle: B lines (on-off effect), c black circle: irregular pleural line, $\mathbf{d}$ white arrows: sub-pleural consolidation, e white arrow: halo sign (Fig. 1, same patient), $\mathbf{f}$ white star: pleural effusion, white arrows: consolidations
Table 3 CT and LUS score

\begin{tabular}{lrrl}
\hline & CT score & LUS score & \multicolumn{1}{l}{$P^{* / * *}$} \\
\hline Total & $14.5 \pm 5.3$ & $19.9 \pm 7.6$ & $<\mathbf{0 . 0 0 1} / r: 0.857$ \\
Right $(n=58)$ & $5 \pm 2.5$ & $10.6 \pm 4.4$ & $0.113 / r: 0.207$ \\
Left $(n=56)$ & $9.3 \pm 3.6$ & $9 \pm 5.4$ & $<\mathbf{0 . 0 0 1} / r: 0.551$ \\
\hline
\end{tabular}

Mean \pm SD. Spearman's rho correlation test was used

*Correlation coefficient, $* * P$ value

artifacts created by the combined B lines, which they said could be observed in the early phase of the ground-glass image found on CT (on-off effect) [19]. In another study that found different B line abnormalities (focal, multifocal, confluent) in patients with COVID-19 pneumonia, it was mentioned that the reversed halo sign seen on CT was not observed in SARS and MERS and could be specific to COVID-19 [20]. A study in which LUS was performed in

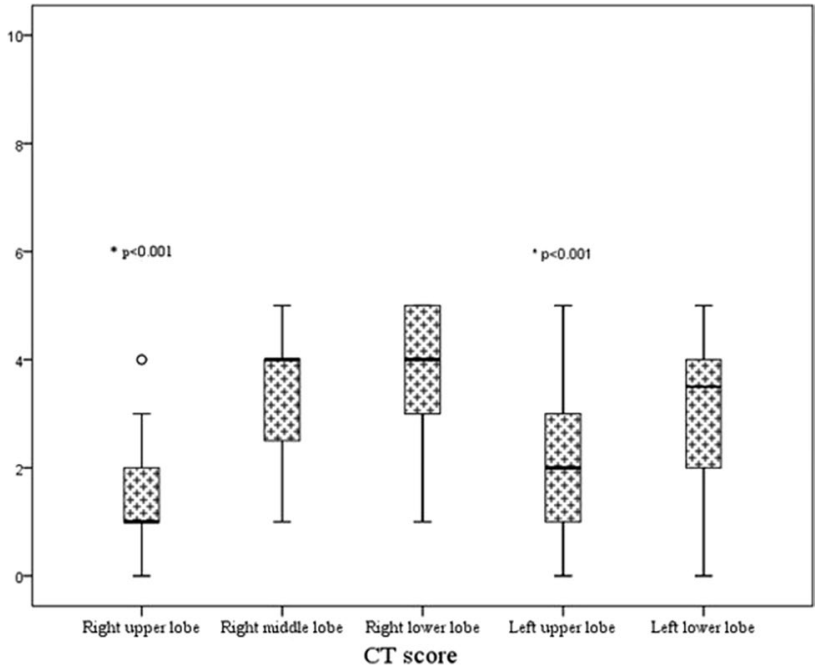

Fig. 3 The CT scores of lobes 
Table 4 Comparison of $\mathrm{CT}$ and LUS findings

\begin{tabular}{lll}
\hline CT findings $(n: 720)$ & LUS findings & \\
\hline Ground-glass opacities $n: 502(\% 82)$ & Irregular pleural line & $222(\% 37)$ \\
& Compound B lines (less than 50\% of the & $201(\% 33)$ \\
& $\quad$ intercostal space) & \\
& Diffuse B lines (white lung) & $169(\% 28)$ \\
Crazy paving sign $n: 86(\% 11.9)$ & Irregular pleural line & $26(\% 30.3)$ \\
& Compound B lines (less than 50\% of the & $25(\% 29.7)$ \\
& intercostal space) & \\
Consolidation $n: 6(\% 0.8)$ & Diffuse B lines (white lung) & $25(\% 40)$ \\
& Consolidation & $6(\% 100)$ \\
\hline
\end{tabular}

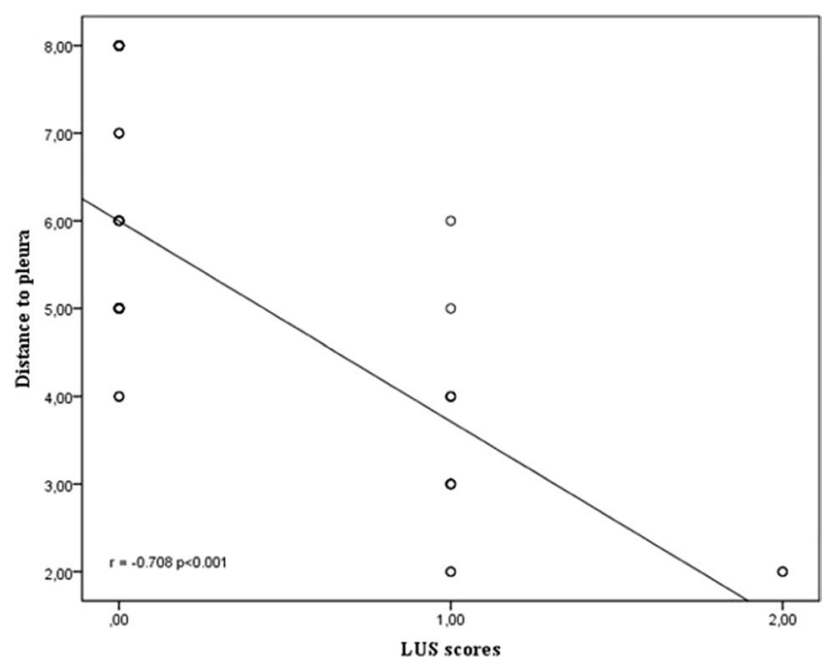

Fig. 4 Correlation between the measured distance to the pleura and the LUS scores

12 regions using a technique similar to that in our study yielded an LUS score of 10.6. In that study, which was found to be perfectly correlated with $\mathrm{CT}$, the visual findings were intense $\mathrm{B}$ line and pleural line abnormalities, similar to other studies [21].

Even though there was positive feedback on the use of LUS for the diagnosis of COVID-19 pneumonia in these studies, there were also studies that highlighted the limitations of LUS. In a study by Huang et al., the presence of continuous or discontinuous B lines and absence of A lines on LUS in COVID-19 patients were reported, which were similar to other results in the literature. They found a higher probability of B line fusion and fixation compared to cardiogenic pulmonary edema. Moreover, they stated that LUS could not substitute for CT and should be used as an additional method for diagnosis [22].

In another study involving 26 patients, they found that CT scores and LUS scores were highly correlated and could be used in diagnosis. They stated that although LUS scores were found to be higher in patients with diffuse ground-glass opacification than those with patchy groundglass opacification, CT may be required to classify the severity of (lung) involvement. They surmised that pleural line abnormalities and B line abnormalities could be associated with idiopathic / pulmonary fibrosis, and that diffuse $B$ line findings could also be seen in treatment-responsive pulmonary congestion [23]. The fact that the researchers used different techniques (number of areas scanned, probe type used, LUS scoring method) during LUS limits the possibility of discussion. Studies have found excellent consistency with CT results via different methods of diagnosis, and they seem to agree on B line and pleural line abnormalities, which are dominant findings.

Our study results yielded satisfactory LUS scores with $\mathrm{CT}$, similar to the results of the studies in the literature. The abnormalities of the B lines on LUS and pleural line abnormalities, which are frequently seen in the literature, are also included in the results of this study. In contrast, the results of this study were that CT lesions did not cause a single finding on LUS, and the ground-glass opacification appearance could lead to combined/continuous B lines and pleural irregularities. It supports the idea that the intensity of the opacification may affect the B line morphology on LUS [19-23]. While the rate of detecting pleural effusion was observed in only two patients (one patient bilateral) in our study, it was obtained at higher rates in different studies [22, 23]. We assume that the density of the lesions and the stage of the disease may be effective in this situation. Although a high correlation was found between thoracic CT scores and LUS scores in our study and in the literature, there are some reservations about the use of LUS in pneumonia. The negative correlation between the contact and distance to the pleura of the lesions identified in thoracic CT, which we evaluated within the context of our study, and the LUS findings supports these concerns [15]. LUS was poor at defining lesions without contact with the pleura, and close lesions that were not in contact with the pleura could cause pleural line abnormalities, whereas $70.5 \%$ of the lesions could never be identified. 


\section{Limitations}

Except for the fact that only the convex probe was used and the LUS evaluations were not repeated, hospitalization of the patients in the intensive care unit might have caused versatility in establishing a diagnosis for researchers. In addition, the main disadvantages of ultrasonography, such as the technical features of ultrasonography and user-dependent variables, are among the limitations of our study.

\section{Conclusion}

Under the current circumstances, LUS seems to be usable as an auxiliary method in establishing the diagnosis with different techniques. We believe that the difficulty in defining lesions not related to the pleura is a disadvantage of LUS. To better assess the lesion morphology, it may be necessary to combine convex and linear probes and to examine pleural lesions better. The fact that LUS scoring and assessment techniques are not standardized makes comparisons among researchers difficult. On the other hand, the advantages of ultrasonography compared to CT include portability, no exposure to radiation (pregnant women and patients with extra risk for radiation use), low cost, no need for auxiliary personnel, and repeatability [24].

Acknowledgements We would like to express our respect and gratitude to Yavuz Durmuş, M.D, head of the radiology department of our hospital, who died due to COVID-19.

\section{Compliance with ethical standards}

Conflict of interest There are no financial or other relations that could lead to a conflict of interest.

Informed consent Informed consent was obtained from all individual participants included in the study.

Ethical approval All procedures performed in studies involving human participants were in accordance with the ethical standards of the institutional and/or national research committee and with the 1964 Helsinki Declaration and its later amendments or comparable ethical standards.

\section{References}

1. Cucinotta D, Vanelli M. WHO declares COVID-19 a pandemic. Acta Biomed. 2020;91:157-60.

2. Wujtewicz M, Dylczyk-Sommer A, Aszkiełowicz A, et al. COVID-19-what should anaethesiologists and intensivists know about it? Anaesthesiol Intensive Ther. 2020;52:34-41.

3. Chung M, Bernheim A, Mei X, et al. CT imaging features of 2019 novel coronavirus (2019-nCoV). Radiology. 2020;295:202-7.
4. Guan WJ, Ni ZY, Hu Y, et al. China Medical Treatment Expert Group for COVID-19. Clinical characteristics of coronavirus disease 2019 in China. N Engl J Med. 2020;382:1708-20.

5. Ai T, Yang Z, Hou H, et al. Correlation of chest CT and RT-PCR testing for coronavirus disease 2019 (COVID-19) in China: a report of 1014 cases. Radiology. 2020;296:32-40.

6. Zhao L, Yu K, Zhao Q, et al. Lung ultrasound score in evaluating the severity of coronavirus disease 2019 (COVID-19) pneumonia. Ultrasound Med Biol. 2020;46:2938-44.

7. Long L, Zhao HT, Zhang ZY, et al. Lung ultrasound for the diagnosis of pneumonia in adults: a meta-analysis. Medicine (Baltimore). 2017;96:e5713.

8. Lichtenstein DA, Meziere GA. Relevance of lung ultrasound in the diagnosis of acute respiratory failure: the BLUE protocol. Chest. 2008;134:117-25.

9. Smith MJ, Hayward SA, Innes SM, et al. Point-of-care lung ultrasound in patients with COVID-19-a narrative review. Anaesthesia. 2020;75:1096-104.

10. Chang YC, Yu CJ, Chang SC, et al. Pulmonary sequelae in convalescent patients after severe acute respiratory syndrome: evaluation with thin-section CT. Radiology. 2005;236:1067-75.

11. Prokop M, van Everdingen W, van Rees Vellinga T, et al. COVID-19 Standardized Reporting Working Group of the Dutch Radiological Society. CO-RADS: a categorical CT assessment scheme for patients suspected of having COVID19-definition and evaluation. Radiology. 2020;296:E97-104.

12. Soummer A, Perbet S, Brisson H, et al. Lung Ultrasound Study Group. Ultrasound assessment of lung aeration loss during a successful weaning trial predicts postextubation distress. Crit Care Med. 2012;40:2064-72.

13. Rouby JJ, Arbelot C, Gao Y, et al. Training for lung ultrasound score measurement in critically ill patients. Am J Respir Crit Care Med. 2018;198:398-401.

14. Lichtenstein DA, Lascols N, Mezière G, et al. Ultrasound diagnosis of alveolar consolidation in the critically ill. Intensive Care Med. 2004;30:276-81.

15. Reissig A, Copetti R, Mathis G, et al. Lung ultrasound in the diagnosis and follow-up of community-acquired pneumonia: a prospective, multicenter, diagnostic accuracy study. Chest. 2012;142:965-72.

16. Sperandeo M, Carnevale V, Muscarella S, et al. Clinical application of transthoracic ultrasonography in inpatients with pneumonia. Eur J Clin Invest. 2011;41:1-7.

17. Liu XL, Lian R, Tao YK, et al. Lung ultrasonography: an effective way to diagnose community-acquired pneumonia. Emerg Med J. 2015;32:433-8.

18. Testa A, Soldati G, Copetti R, et al. Early recognition of the 2009 pandemic influenza A (H1N1) pneumonia by chest ultrasound. Crit Care. 2012;16:R30.

19. Volpicelli G, Lamorte A, Villén T. What's new in lung ultrasound during the COVID-19 pandemic. Intensive Care Med. 2020;46:1445-8.

20. Lomoro P, Verde F, Zerboni F, et al. COVID-19 pneumonia manifestations at the admission on chest ultrasound, radiographs, and CT: single-center study and comprehensive radiologic literature review. Eur J Radiol Open. 2020;7:100231.

21. Tung-Chen Y, Martí de Gracia M, Díez-Tascón A, et al. Correlation between chest computed tomography and lung ultrasonography in patients with coronavirus disease 2019 (COVID-19). Ultrasound Med Biol. 2020;46:2918-26.

22. Huang Y, Wang S, Liu Y, et al. A preliminary study on the ultrasonic manifestations of peripulmonary lesions of non-critical novel coronavirus pneumonia (COVID-19) . SSRN. 2020. https ://doi.org/10.2139/ssrn.3544750. 
23. Nouvenne A, Zani MD, Milanese G, et al. Lung ultrasound in COVID-19 pneumonia: correlations with chest CT on hospital admission. Respiration. 2020;99:617-24.

24. Buonsenso D, Piano A, Raffaelli F, et al. Point-of-care lung ultrasound findings in novel coronavirus disease-19 pneumonia: a case report and potential applications during COVID-19 outbreak. Eur Rev Med Pharmacol Sci. 2020;24:2776-80.
Publisher's Note Springer Nature remains neutral with regard to jurisdictional claims in published maps and institutional affiliations. 